\title{
Index theorems for meromorphic self-maps of the projective space
}

\author{
Marco Abate \\ Dipartimento di Matematica, Università di Pisa \\ Largo Pontecorvo 5, 56127 Pisa, Italy. \\ E-mail: abate@dm.unipi.it
}

June 2011

\section{Dedicated to Jack Milnor for his 80th birthday}

\begin{abstract}
In this short note we would like to show how it is possible to use techniques introduced in the theory of local dynamics of holomorphic germs tangent to the identity to study global meromorphic self-maps of the complex projective space. In particular we shall show how a meromorphic map $f: \mathbb{P}^{n} \rightarrow \mathbb{P}^{n}$ induces a holomorphic foliation of $\mathbb{P}^{n}$ in Riemann surfaces, whose singular points are exactly the fixed points and the indeterminacy points of $f$; and we shall prove three index theorems, relating suitably defined local residues at the fixed and indeterminacy points of $f$ with Chern classes of $\mathbb{P}^{n}$.
\end{abstract}

In this short note we would like to show how the techniques introduced in [ABT1] (see also [ABT2, 3], and [AT2]) for studying the local dynamics of holomorphic germs tangent to the identity can be used to study global meromorphic self-maps of the complex projective space $\mathbb{P}^{n}$. More precisely, we shall prove the following index theorem:

Theorem 1: Let $f: \mathbb{P}^{n} \rightarrow \mathbb{P}^{n}$ be a meromorphic self-map of degree $\nu+1 \geq 2$ of the complex $n$-dimensional projective space. Let $\Sigma(f)=\operatorname{Fix}(f) \cup I(f)$ be the union of the indeterminacy set $I(f)$ of $f$ and the fixed points set $\operatorname{Fix}(f)$ of $f$. Let $\Sigma(f)=\sqcup_{\alpha} \Sigma_{\alpha}$ be the decompositon of $\Sigma$ in connected components, and denote by $N$ the tautological line bundle of $\mathbb{P}^{n}$. Then:

(i) we can associate to each $\Sigma_{\alpha}$ a complex number $\operatorname{Res}^{1}\left(f, \Sigma_{\alpha}\right) \in \mathbb{C}$, depending only on the local behavior of $f$ nearby $\Sigma_{\alpha}$, so that

$$
\sum_{\alpha} \operatorname{Res}^{1}\left(f, \Sigma_{\alpha}\right)=\int_{\mathbb{P}^{n}} c_{1}(N)^{n}=(-1)^{n}
$$

(ii) given a homogeneous symmetric polynomial $\varphi \in \mathbb{C}\left[z_{1}, \ldots, z_{n}\right]$ of degree $n$ we can associate to each $\Sigma_{\alpha}$ a complex number $\operatorname{Res}_{\varphi}^{2}\left(f, \Sigma_{\alpha}\right) \in \mathbb{C}$, depending only on the local behavior of $f$ nearby $\Sigma_{\alpha}$, such that

$$
\sum_{\alpha} \operatorname{Res}_{\varphi}^{2}\left(f, \Sigma_{\alpha}\right)=\int_{\mathbb{P}^{n}} \varphi\left(T \mathbb{P}^{n}-N^{\otimes \nu}\right) ;
$$

(iii) if $\nu>1$, given a homogeneous symmetric polynomial $\psi \in \mathbb{C}\left[z_{0}, \ldots, z_{n}\right]$ of degree $n$ we can associate to each $\Sigma_{\alpha}$ a complex number $\operatorname{Res}_{\psi}^{3}\left(f, \Sigma_{\alpha}\right) \in \mathbb{C}$, depending only on the local behavior of $f$ nearby $\Sigma_{\alpha}$, such that

$$
\sum_{\alpha} \operatorname{Res}_{\psi}^{3}\left(f, \Sigma_{\alpha}\right)=\int_{P^{n}} \psi\left(\left(T \mathbb{P}^{n} \oplus N\right)-N^{\otimes \nu}\right) .
$$

In this statement, if $\varphi \in \mathbb{C}\left[z_{1}, \ldots, z_{r}\right]$ is a homogeneous symmetric polynomial and $E$ and $F$ are vector bundles over $\mathbb{P}^{n}$, we put

$$
\varphi(E-F)=\tilde{\varphi}\left(c_{1}(E-F), \ldots, c_{r}(E-F)\right),
$$

where the $c_{j}(E-F)$ are the Chern classes of the virtual bundle $E-F$, and $\tilde{\varphi} \in \mathbb{C}\left[z_{1}, \ldots, z_{r}\right]$ is the unique polynomial such that

$$
\varphi=\tilde{\varphi}\left(\sigma_{1}, \ldots, \sigma_{r}\right)
$$

where $\sigma_{1}, \ldots, \sigma_{r} \in \mathbb{C}\left[z_{1}, \ldots, z_{r}\right]$ are the elementary symmetric functions on $n$ variables.

2010 Mathematics Subject Classification: Primary 32H04; 32H50, 14M20, 37F75, 37F99, 58J20 
Remark 1: If $\Sigma(f)$ is finite, then the number of points in $\Sigma(f)$, counted with respect to a suitable multiplicity, is

$$
\frac{1}{\nu}\left[(\nu+1)^{n+1}-1\right]=\sum_{k=0}^{n}\left(\begin{array}{l}
n+1 \\
k+1
\end{array}\right) \nu^{k}
$$

see, e.g., [AT1].

Remark 2: Since the total Chern classes of $T \mathbb{P}^{n}$ in terms of the first Chern class of $N$ is given by $c\left(T \mathbb{P}^{n}\right)=\left(1-c_{1}(N)\right)^{n+1}$, the total Chern class of $T \mathbb{P}^{n}-N^{\otimes \nu}$ is given by

$$
c\left(T \mathbb{P}^{n}-N^{\otimes \nu}\right)=\frac{\left(1-c_{1}(N)\right)^{n+1}}{1+\nu c_{1}(N)}=\left(1-c_{1}(N)\right)^{n+1} \sum_{j=0}^{n}(-1)^{j} \nu^{j} c_{1}(N)^{j}
$$

and thus the Chern classes of $T \mathbb{P}^{n}-N^{\otimes \nu}$ are given by

$$
c_{j}\left(T \mathbb{P}^{n}-N^{\otimes \nu}\right)=(-1)^{j} \sum_{k=0}^{j}\left(\begin{array}{c}
n+1 \\
k
\end{array}\right) \nu^{j-k} c_{1}^{j}(N) .
$$

In particular,

$$
\int_{\mathbb{P}^{n}} c_{n}\left(T \mathbb{P}^{n}-N^{\otimes \nu}\right)=\sum_{k=0}^{n}\left(\begin{array}{c}
n+1 \\
k
\end{array}\right) \nu^{n-k} \quad \text { and } \quad \int_{\mathbb{P}^{n}} c_{1}^{n}\left(T \mathbb{P}^{n}-N^{\otimes \nu}\right)=(\nu+1+n)^{n} .
$$

Arguing in a similar way we get that the Chern classes of $\left(T \mathbb{P}^{n} \oplus N\right)-N^{\otimes \nu}$ are given by

$$
c_{j}\left(\left(T \mathbb{P}^{n} \oplus N\right)-N^{\otimes \nu}\right)=(-1)^{j} \sum_{k=0}^{j}\left[\left(\begin{array}{c}
n+1 \\
k
\end{array}\right)-\left(\begin{array}{c}
n+1 \\
k-1
\end{array}\right)\right] \nu^{j-k} c_{1}^{j}(H),
$$

with the convention $\left(\begin{array}{c}n+1 \\ -1\end{array}\right)=0$.

The usefulness of a theorem like Theorem 1 is of course related to the possibility of computing the residues involved, and indeed we have explicit formulas for the (generic) case of point components, expressed in terms of Grothendieck residues (the residues at components of positive dimension can be expressed by using Lehmann-Suwa theory; see [LS], [S, Chapter IV] and [ABT1, (6.1)]). To state the formulas we recall three definitions.

Definition 1: Let $\mathcal{O}_{w^{o}}^{n}$ be the ring of germs of holomorphic functions at $w^{o} \in \mathbb{C}^{n}$. Take $g_{1}, \ldots, g_{n} \in \mathcal{O}_{w^{o}}^{n}$ such that $w^{o}$ is an isolated zero of $g=\left(g_{1}, \ldots, g_{n}\right)$. Then the Grothendieck residue of $h \in \mathcal{O}_{w^{o}}^{n}$ along $g_{1}, \ldots, g_{n}$ is defined (see $[\mathrm{H}]$, [L, Section 5], [LS, Section 4], [S, pp.105-107]) by the formula

$$
\operatorname{Res}_{w^{o}}\left[\begin{array}{c}
h(w) d w_{1} \wedge \cdots \wedge d w_{n} \\
g_{1}, \ldots, g_{n}
\end{array}\right]=\left(\frac{1}{2 \pi i}\right)^{n} \int_{\Gamma} \frac{h}{g_{1} \cdots g_{n}} d w_{1} \wedge \cdots \wedge d w_{n},
$$

where $\Gamma=\left\{\left|g_{j}(w)\right|=\varepsilon \mid j=1, \ldots, n\right\}$ for $\varepsilon>0$ small enough, oriented so that $d \arg \left(g_{1}\right) \wedge \cdots \wedge d \arg \left(g_{n}\right)>0$.

Definition 2: Let $\varphi \in \mathbb{C}\left[z_{1}, \ldots, z_{n}\right]$ be a homogeneous symmetric polynomial in $n$ variables, and $L: V \rightarrow V$ an endomorphism of an $n$-dimensional complex vector space $V$. Then we set

$$
\varphi(L)=\varphi\left(\lambda_{1}, \ldots, \lambda_{n}\right),
$$

where $\lambda_{1}, \ldots, \lambda_{n} \in \mathbb{C}$ are the eigenvalues of $L$.

Definition 3: We shall say that a homogeneous polynomial self-map $F=\left(F_{0}, \ldots, F_{n}\right): \mathbb{C}^{n+1} \rightarrow \mathbb{C}^{n+1}$ of degree $\nu+1$ induces the meromorphic self-map $f: \mathbb{P}^{n} \rightarrow \mathbb{P}^{n}$ if

$$
f\left(\left[z_{0}: \cdots: z_{n}\right]\right)=\left[F_{0}\left(z_{0}, \ldots, z_{n}\right): \cdots: F_{n}\left(z_{0}, \ldots, z_{n}\right)\right]
$$

for all $\left[z_{0}: \cdots: z_{n}\right] \in \mathbb{P}^{n}$. It is well known (see, e.g., [FS]) that every meromorphic self-map of $\mathbb{P}^{n}$ of degree $\nu+1$ is induced by a unique homogeneous polynomial self-map of $\mathbb{C}^{n+1}$ of degree $\nu+1$.

We can now state our next theorem: 
Theorem 2: Let $f: \mathbb{P}^{n} \rightarrow \mathbb{P}^{n}$ be a meromorphic self-map of degree $\nu+1 \geq 2$ of the complex $n$-dimensional projective space. Let $\Sigma(f)=\operatorname{Fix}(f) \cup I(f)$ be the union of the indeterminacy set $I(f)$ of $f$ and the fixed points set $\operatorname{Fix}(f)$ of $f$, and assume that $p=\left[1: w_{1}^{o}: \cdots: w_{n}^{o}\right]$ is an isolated point in $\Sigma(f)$. Set $w^{o}=\left(w_{1}^{o}, \ldots, w_{n}^{o}\right)$, let $F=\left(F_{0}, \ldots, F_{n}\right): \mathbb{C}^{n+1} \rightarrow \mathbb{C}^{n+1}$ be the homogeneous polynomial self-map of degree $\nu+1$ inducing $f$, and define $g: \mathbb{C}^{n} \rightarrow \mathbb{C}^{n}$ by setting

$$
g_{j}(w)=F_{j}(1, w)-w_{j} F_{0}(1, w)
$$

for all $w \in \mathbb{C}^{n}$ and $j=1, \ldots, n$. Then:

(i) we have

$$
\operatorname{Res}^{1}(f, p)=\operatorname{Res}_{w^{o}}\left[\begin{array}{c}
F_{0}(1, w)^{n} d w_{1} \wedge \cdots \wedge d w_{n} \\
g_{1}, \ldots, g_{n}
\end{array}\right]
$$

(ii) if $\varphi \in \mathbb{C}\left[z_{1}, \ldots, z_{n}\right]$ is a homogeneous symmetric polynomial of degree $n$ then

$$
\operatorname{Res}_{\varphi}^{2}(f, p)=\operatorname{Res}_{w^{o}}\left[\begin{array}{c}
\varphi\left(d g_{w}\right) d w_{1} \wedge \cdots \wedge d w_{n} \\
g_{1}, \ldots, g_{n}
\end{array}\right]
$$

(iii) if $\psi \in \mathbb{C}\left[z_{0}, \ldots, z_{n}\right]$ is a homogeneous symmetric polynomial of degree $n$ then

$$
\operatorname{Res}_{\psi}^{3}(f, p)=\operatorname{Res}_{w^{o}}\left[\begin{array}{c}
\psi\left(F_{0}(1, w), \mu_{1}(w), \ldots, \mu_{n}(w)\right) d w_{1} \wedge \cdots \wedge d w_{n} \\
g_{1}, \ldots, g_{n}
\end{array}\right],
$$

where $\mu_{1}(w), \ldots, \mu_{n}(w)$ are the eigenvalues of $d g_{w}$.

This statement is effective because we can explicitly compute a Grothendieck residue using an algorithm suggested by Hartshorne (see $[\mathrm{BB}]$ and $[\mathrm{H}]$ ). Without loss of generality, we can assume $w^{o}=O$. Since the origin is an isolated zero of $g$, there exist minimal positive integers $\alpha_{1}, \ldots, \alpha_{n}$ such that $w_{1}^{\alpha_{1}}, \ldots, w_{n}^{\alpha_{n}}$ belong to the ideal generated by $g_{1}, \ldots, g_{n}$ in $\mathcal{O}^{n}=\mathcal{O}_{O}^{n}$. Hence there exist holomorphic functions $b_{i j} \in \mathcal{O}^{n}$ such that

$$
w_{i}^{\alpha_{i}}=\sum_{j=1}^{n} b_{i j} g_{j} .
$$

The properties of the Grothendieck residue (see $[\mathrm{H}])$ then imply

$$
\operatorname{Res}_{O}\left[\begin{array}{c}
h d w_{1} \wedge \cdots \wedge d w_{n} \\
g_{1}, \cdots, g_{n}
\end{array}\right]=\operatorname{Res}_{O}\left[\begin{array}{c}
h \operatorname{det}\left(b_{i j}\right) d w_{1} \wedge \cdots \wedge d w_{n} \\
w_{1}^{\alpha_{1}}, \cdots, w_{n}^{\alpha_{n}}
\end{array}\right]
$$

The right-hand side is now evaluated by expanding $h \operatorname{det}\left(b_{i j}\right)$ in a power series in the $w_{i}$; the residue is given by the coefficient of $w_{1}^{\alpha_{1}-1} \cdots w_{n}^{\alpha_{n}-1}$.

The easiest case is when $\operatorname{det}\left(d g_{O}\right) \neq 0$. Indeed, since $O$ is an isolated zero of $g$ we can write

$$
g_{j}=\sum_{i=1}^{k} c_{j i} w_{i} .
$$

Differentiating this and evaluating in $O$ we get

$$
\frac{\partial g_{j}}{\partial w_{k}}(O)=c_{j k}(O)
$$

therefore if $\operatorname{det}\left(d g_{O}\right) \neq 0$ we can invert the matrix $\left(c_{j i}\right)$ in a neighborhood of $O$ and write

$$
w_{i}=\sum_{j=1}^{n} b_{i j} g_{j}
$$

where $\left(b_{i j}\right)$ is the inverse matrix of $\left(c_{j i}\right)$. So we have $\alpha_{1}=\cdots=\alpha_{n}=1$ and $\operatorname{det}\left(b_{i j}\right)(O)=1 / \operatorname{det}\left(d g_{O}\right)$. 
It thus follows that

$$
\operatorname{det}\left(d g_{w^{o}}\right) \neq 0 \Longrightarrow \operatorname{Res}_{w^{o}}\left[\begin{array}{c}
h(w) d w_{1} \wedge \cdots \wedge d w_{n} \\
g_{1}, \ldots, g_{n}
\end{array}\right]=\frac{h\left(w^{o}\right)}{\mu_{1} \cdots \mu_{n}}
$$

where $\mu_{1}, \ldots, \mu_{n} \in \mathbb{C}$ are the eigenvalues of $d g_{w^{o}}$.

In our situation, $g_{j}(w)=F_{j}(1, w)-w_{j} F_{0}(1, w)$; therefore we have

$$
\frac{\partial g_{j}}{\partial w_{k}}(w)=\frac{\partial F_{j}}{\partial w_{k}}(1, w)-w_{j} \frac{\partial F_{0}}{\partial w_{k}}(1, w)-\delta_{k}^{j} F_{0}(1, w),
$$

where $\delta_{k}^{j}$ is Kronecker's delta.

So if $p=\left[1: w_{1}^{o}: \cdots: w_{n}^{o}\right]$ is an indeterminacy point we have $F_{0}\left(1, w^{o}\right)=0$ and

$$
\operatorname{Jac}(g)\left(w^{o}\right)=\left(\frac{\partial F_{j}}{\partial w_{k}}\left(1, w^{o}\right)-w_{j}^{o} \frac{\partial F_{0}}{\partial w_{k}}\left(1, w^{o}\right)\right)_{j, k=1, \ldots, n}
$$

If instead $p$ is a fixed point, we can consider the differential of $f$ at $p$. Let $\chi$ be the usual chart of $\mathbb{P}^{n}$ centered at $[1: 0: \cdots: 0]$. Then

$$
\tilde{f}(w)=\chi \circ f \circ \chi^{-1}(w)=\left(\frac{F_{1}(1, w)}{F_{0}(1, w)}, \ldots, \frac{F_{n}(1, w)}{F_{0}(1, w)}\right)
$$

and so

$$
\frac{\partial \tilde{f}_{j}}{\partial w_{k}}(w)=\frac{1}{F_{0}(1, w)}\left[\frac{\partial F_{j}}{\partial w_{k}}(1, w)-\frac{F_{j}(1, w)}{F_{0}(1, w)} \frac{\partial F_{0}}{\partial w_{k}}(1, w)\right] .
$$

In particular,

$$
\frac{\partial \tilde{f}_{h}}{\partial w_{k}}\left(w^{o}\right)=\frac{1}{F_{0}\left(1, w^{o}\right)}\left[\frac{\partial F_{j}}{\partial w_{k}}\left(1, w^{o}\right)-w_{j}^{o} \frac{\partial F_{0}}{\partial w_{k}}\left(1, w^{o}\right)\right]
$$

therefore

$$
\operatorname{Jac}(g)\left(w^{o}\right)=F_{0}\left(1, w^{o}\right)\left[\operatorname{Jac}(\tilde{f})\left(w^{o}\right)-I\right]
$$

It follows that the eigenvalues $\mu_{1}, \ldots, \mu_{n}$ of $d g_{w^{o}}$ are related to the eigenvalues $\lambda_{1}, \ldots, \lambda_{n}$ of $d f_{p}$ by the formula

$$
\forall j=1, \ldots, n \quad \mu_{j}=F_{0}\left(1, w^{o}\right)\left(\lambda_{j}-1\right) .
$$

In particular, $\operatorname{det}\left(d g_{w^{o}}\right) \neq 0$ if and only if 1 is not an eigenvalue of $d f_{p}$, that is if and only if $p$ is a simple fixed point of $f$.

Summing up, these computations give the following particular case of Theorem 2:

Corollary 3: Let $f: \mathbb{P}^{n} \rightarrow-\rightarrow \mathbb{P}^{n}$ be a meromorphic self-map of degree $\nu+1 \geq 2$ of the complex $n$-dimensional projective space.

(a) Assume that $p \in \mathbb{P}^{n}$ is a simple (necessarily isolated) fixed point of $f$, and let $\lambda_{1}, \ldots, \lambda_{n} \neq 1$ be the eigenvalues of $d f_{p}$. Then:

(i) we have

$$
\operatorname{Res}^{1}(f, p)=\frac{(-1)^{n}}{\left(1-\lambda_{1}\right) \cdots\left(1-\lambda_{n}\right)}
$$

(ii) if $\varphi \in \mathbb{C}\left[z_{1}, \ldots, z_{n}\right]$ is a homogeneous symmetric polynomial of degree $n$ then

$$
\operatorname{Res}_{\varphi}^{2}(f, p)=\frac{\varphi\left(1-\lambda_{1}, \ldots, 1-\lambda_{n}\right)}{\left(1-\lambda_{1}\right) \cdots\left(1-\lambda_{n}\right)}
$$


(iii) if $\psi \in \mathbb{C}\left[z_{0}, \ldots, z_{n}\right]$ is a homogeneous symmetric polynomial of degree $n$ then

$$
\operatorname{Res}_{\psi}^{3}(f, p)=(-1)^{n} \frac{\psi\left(1, \lambda_{1}-1, \ldots, \lambda_{n}-1\right)}{\left(1-\lambda_{1}\right) \cdots\left(1-\lambda_{n}\right)}
$$

(b) Assume that $p=\left[1: w_{1}^{o}: \cdots: w_{n}^{o}\right]$ is an isolated indeterminacy point, and that $\operatorname{det} G \neq 0$, where

$$
G=\left(\frac{\partial F_{j}}{\partial w_{k}}\left(1, w^{o}\right)-w_{j}^{o} \frac{\partial F_{0}}{\partial w_{k}}\left(1, w^{o}\right)\right)_{j, k=1, \ldots, n},
$$

and $F=\left(F_{0}, \ldots, F_{n}\right): \mathbb{C}^{n+1} \rightarrow \mathbb{C}^{n+1}$ is the homogeneous polynomial self-map of degree $\nu+1$ inducing $f$. Denote by $\mu_{1}, \ldots, \mu_{n} \neq 0$ the eigenvalues of $G$. Then:

(i) we have

$$
\operatorname{Res}^{1}(f, p)=0 ;
$$

(ii) if $\varphi \in \mathbb{C}\left[z_{1}, \ldots, z_{n}\right]$ is a homogeneous symmetric polynomial of degree $n$ then

$$
\operatorname{Res}_{\varphi}^{2}(f, p)=\frac{\varphi\left(\mu_{1}, \ldots, \mu_{n}\right)}{\mu_{1} \cdots \mu_{n}}
$$

(iii) if $\psi \in \mathbb{C}\left[z_{0}, \ldots, z_{n}\right]$ is a homogeneous symmetric polynomial of degree $n$ then

$$
\operatorname{Res}_{\psi}^{3}(f, p)=\frac{\psi\left(0, \mu_{1}, \ldots, \mu_{n}\right)}{\mu_{1} \cdots \mu_{n}}
$$

This corollary shows that our Theorem 1 is related to Ueda's index theorem, which however applies only to holomorphic self-maps having only simple fixed points:

Theorem 4: (Ueda [U]) Let $f: \mathbb{P}^{n} \rightarrow \mathbb{P}^{n}$ be holomorphic of degree $\nu+1 \geq 2$, and assume that all fixed points of $f$ are simple (and thus isolated). For $p \in \operatorname{Fix}(f)$, let $\lambda_{1}(p), \ldots, \lambda_{n}(p) \neq 1$ denote the eigenvalues of $d f_{p}$. Then

$$
\forall k=0, \ldots, n \quad \sum_{p \in \operatorname{Fix}(f)} \frac{\sigma_{k}\left(d f_{p}\right)}{\left(1-\lambda_{1}(p)\right) \cdots\left(1-\lambda_{n}(p)\right)}=(-1)^{k}(\nu+1)^{k} .
$$

For instance, the case $k=0$ is a consequence of Theorem 1.(i), and the cases $k>0$ can be linked to Theorem 1.(ii) and (iii) by using the formula

$$
\sigma_{j}(I-L)=\sum_{\ell=0}^{j}\left(\begin{array}{l}
n-\ell \\
n-j
\end{array}\right)(-1)^{\ell} \sigma_{\ell}(L)
$$

valid for every endomorphism $L$ of an $n$-dimensional complex vector space.

Example 1: Let us consider the map $f([z])=\left[z_{0}^{\nu+1}: \cdots: z_{n}^{\nu+1}\right]$. This map is holomorphic, and all its fixed points are simple. More precisely, for each $\ell=0, \ldots, n$ the set $\operatorname{Fix}(f)$ contains exactly $\left(\begin{array}{l}n+1 \\ \ell+1\end{array}\right) \nu^{\ell}$ fixed points of the form $\left[\zeta_{0}: \cdots: \zeta_{n}\right]$, where $\ell+1$ of the $\zeta_{j}$ are $\nu$-th roots of unity, and the remaining $\zeta_{j}$ 's are equal to 0 . If $p \in \operatorname{Fix}(f)$ has exactly $\ell+1$ non-zero homogeneous coordinates, we shall say that $p$ is a fixed point of level $\ell$.

Using (4) it is easy to see that if $p \in \operatorname{Fix}(f)$ is a fixed point of level $\ell$ then the eigenvalues of $d f_{p}$ are $\nu+1$ with multiplicity $\ell$ and 0 with multiplicity $n-\ell$. In particular, $\left(1-\lambda_{1}(p)\right) \cdots\left(1-\lambda_{n}(p)\right)=(-1)^{\ell} \nu^{\ell}$, and so Theorem 1.(i) becomes

$$
(-1)^{n} \sum_{\ell=0}^{n}\left(\begin{array}{l}
n+1 \\
\ell+1
\end{array}\right)(-1)^{\ell}=(-1)^{n}
$$


while Theorem 1.(ii) becomes

$$
(-1)^{n} \sum_{\ell=0}^{n}\left(\begin{array}{c}
n+1 \\
\ell+1
\end{array}\right)(-1)^{\ell} \varphi(\underbrace{\nu, \ldots, \nu}_{\ell \text { times }},-1, \ldots,-1)=\int_{\mathbb{P}^{n}} \varphi\left(T \mathbb{P}^{n}-N^{\otimes \nu}\right),
$$

and Theorem 1.(iii) becomes

$$
(-1)^{n} \sum_{\ell=0}^{n}\left(\begin{array}{c}
n+1 \\
\ell+1
\end{array}\right)(-1)^{\ell} \psi(1, \underbrace{\nu, \ldots, \nu}_{\ell \text { times }},-1, \ldots,-1)=\int_{\mathbb{P}^{n}} \psi\left(\left(T \mathbb{P}^{n} \oplus N\right)-N^{\otimes \nu}\right) .
$$

For instance, taking $\varphi=\sigma_{1}^{n}$ and recalling (1) we get

$$
(\nu+1+n)^{n}=\int_{\mathbb{P}^{n}} c_{1}^{n}\left(T \mathbb{P}^{n}-N^{\otimes \nu}\right)=(-1)^{n} \sum_{\ell=0}^{n}\left(\begin{array}{c}
n+1 \\
\ell+1
\end{array}\right)(-1)^{\ell}((\nu+1) \ell-n)^{n} .
$$

The (non-trivial) equality of the left and right-hand sides in this formula can also be proved directly by using Abel's formula

$$
(x+y)^{r}=\sum_{k=0}^{r}\left(\begin{array}{l}
r \\
k
\end{array}\right) x(x-k z)^{k-1}(y+k z)^{r-k}
$$

valid for all $r \in \mathbb{N}$ and $x, y, z \in \mathbb{C}$ with $x \neq 0$ (see, e.g., [K, p. 56]). Indeed we have

$$
\begin{aligned}
\sum_{\ell=0}^{n}\left(\begin{array}{l}
n+1 \\
\ell+1
\end{array}\right) & (-1)^{\ell}((\nu+1) \ell-n)^{n} \\
& =\sum_{\ell=0}^{n}\left(\begin{array}{c}
n+1 \\
\ell+1
\end{array}\right)(n-(\nu+1) \ell)^{\ell}(-n+(\nu+1) \ell)^{n-\ell} \\
& =\sum_{k=1}^{n+1}\left(\begin{array}{c}
n+1 \\
k
\end{array}\right)(n+\nu+1-k(\nu+1))^{k-1}(-n-\nu-1+k(\nu+1))^{n+1-k} \\
& =\frac{1}{n+\nu+1}\left[\sum_{k=0}^{n+1}\left(\begin{array}{c}
n+1 \\
k
\end{array}\right)(n+\nu+1)(n+\nu+1-k(\nu+1))^{k-1}(-n-\nu-1+k(\nu+1))^{n+1-k}\right. \\
& \left.\quad+(-1)^{n}(n+\nu+1)^{n+1}\right] \\
& =(-1)^{n}(\nu+n+1)^{n},
\end{aligned}
$$

thanks to (5) applied with $r=n+1, x=-y=n+\nu+1$ and $z=\nu+1$.

Analogously, taking $\varphi=\sigma_{n}$ we get

$$
\sum_{k=0}^{n}\left(\begin{array}{c}
n+1 \\
k
\end{array}\right) \nu^{n-k}=\int_{\mathbb{P}^{n}} c_{n}\left(T \mathbb{P}^{n}-N^{\otimes \nu}\right)=\sum_{\ell=0}^{n}\left(\begin{array}{c}
n+1 \\
\ell+1
\end{array}\right) \nu^{\ell}=\frac{(\nu+1)^{n+1}-1}{\nu}=\sum_{\ell=0}^{n}(\nu+1)^{\ell},
$$

in agreement with both (1) and Theorem 4.

Let us finally show how to use the local theory developed in [ABT1] and [AT2] to prove Theorems 1 and 2 .

Given a meromorphic $f: \mathbb{P}^{n} \rightarrow \mathbb{P}^{n}$ of degree $\nu+1 \geq 2$, let $F=\left(F_{0}, \ldots, F_{n}\right): \mathbb{C}^{n+1} \rightarrow \mathbb{C}^{n+1}$ be the homogeneous polynomial map of degree $\nu+1$ inducing $f$. First of all, we associate to $F$ the homogeneous vector field

$$
Q=\sum_{j=0}^{n} F_{j} \frac{\partial}{\partial z_{j}}
$$


It is well-known that the time- 1 map $f_{Q}$ of $Q$ is a germ of holomorphic self-map of $\mathbb{C}^{n+1}$ tangent to the identity; furthermore we can write $f_{Q}(z)=z+F(z)+O\left(\|z\|^{v+2}\right)$. In particular, by definition a direction $v \in \mathbb{C}^{n+1} \backslash\{O\}$ is a non-degenerate characteristic direction for $f_{Q}$ if and only if $[v] \in \mathbb{P}^{n}$ is a fixed point of $f$; and it is a degenerate characteristic direction for $f_{Q}$ if and only if $[v]$ is an indeterminacy point of $f$. Furthermore, since $f$ has degree at least 2 then $Q$ is non-dicritical, that is not all directions are characteristic.

Now let $\pi: M \rightarrow \mathbb{C}^{n+1}$ be the blow-up of the origin, and denote by $E=\pi^{-1}(O)$ the exceptional divisor. By construction, $E$ is canonically biholomorphic to $\mathbb{P}^{n}$; furthermore, the blow-up $M$ can be identified with the total space of the normal bundle $N_{E}$ of $E$ in $M$, and $N_{E}$ is isomorphic to the tautological line bundle $N$ on $\mathbb{P}^{n}$.

We can now lift $f_{Q}$ to the blow-up, obtaining (see, e.g., [A]) a germ $\hat{f}_{Q}$ about $E$ of holomorphic self-map of $M$ fixing $E$ pointwise, and we may apply all the machinery developed in [ABT1]. First of all, since $Q$ is non-dicritical then $\hat{f}_{Q}$ is tangential and has order of contact $\nu$ with $E$ ([ABT1, Proposition 1.4]). Then we can define ([ABT1, Proposition 3.1 and Corollary 3.2]) the canonical section $X_{f}$, which is a global holomorphic section of $T E \otimes\left(N_{E}^{*}\right)^{\otimes \nu}$. In local coordinates in $M$ centered at $[1: 0: \cdots: 0] \in E$ we can write

$$
X_{f}=\sum_{j=1}^{n} g_{j} \frac{\partial}{\partial w^{j}} \otimes\left(d w_{0}\right)^{\otimes \nu}
$$

where $g_{1}, \ldots, g_{n}$ are given by (2), and $E$ in these coordinates is given by $\left\{w_{0}=0\right\}$.

In particular, we can think of $X_{f}$ as a bundle morphism $X_{f}: N_{E}^{\otimes \nu} \rightarrow T E$, vanishing exactly on the characteristic directions of $f_{Q}$, and thus we have proved the following

Proposition 5: Let $f: \mathbb{P}^{n} \rightarrow-\rightarrow \mathbb{P}^{n}$ be a meromorphic self-map of degree $\nu+1 \geq 2$. Then the canonical section $X_{f}$, given locally by (6), defines a global singular holomorphic foliation of $\mathbb{P}^{n}$ in Riemann surfaces, singular exactly at the fixed and indeterminacy points of $f$.

We can now apply the index theorems proved in [ABT1]. Theorem 1.(i) follows immediately from the Camacho-Sad-like index Theorem 6.2 in [ABT1], recalling that $\int_{\mathbb{P} n} c_{1}(N)^{n}=(-1)^{n}$. Theorem 1.(ii) follows from the Baum-Bott index theorem (see [S, Th. III.7.6] and [ABT1, Theorem 6.4]); and Theorem 1.(iii) follows from the Lehmann-Suwa-like index Theorem 6.3 in [ABT1], that can be applied to this situation because $E$ is automatically comfortably embedded in $M$ ([ABT1, Example 2.4]), and recalling that the exact sequence

$$
\left.O \longrightarrow T E \longrightarrow T M\right|_{E} \longrightarrow N_{E} \longrightarrow O
$$

implies that $c\left(\left.T M\right|_{E}\right)=c\left(T \mathbb{P}^{n} \oplus N\right)$.

Theorem 2 also follows from [ABT1]. Indeed, Theorem 2.(i) is an immediate consequence of [ABT1, Theorem 6.5.(i)] and the computations in [AT2, Section 5]. The same computations and [ABT1, Theorem 6.6] yield Theorem 2.(iii); and Theorem 2.(ii) follows from [BB] and [S, Th. III.5.5].

Acknowledgments. I would like to thank Francesca Tovena, Jasmin Raissy and Matteo Ruggiero for many useful conversations, Nurìa Fagella and the Institut de Matemàtica de la Universitat de Barcelona for their wonderful hospitality during the preparation of this note, and Jack Milnor for creating so much beautiful mathematics and for being of inspiration to us all. 


\section{References.}

[A] M. Abate: Diagonalization of non-diagonalizable discrete holomorphic dynamical systems. Amer. J. Math. 122 (2000), 757-781.

[ABT1] M. Abate, F. Bracci, F. Tovena: Index theorems for holomorphic self-maps. Ann. of Math. 159 (2004), 819-864.

[ABT2] M. Abate, F. Bracci, F. Tovena: Index theorems for holomorphic maps and foliations. Indiana Univ. Math. J. 57 (2008), 2999-3048.

[ABT3] M. Abate, F. Bracci, F. Tovena: Embeddings of submanifolds and normal bundles. Adv. Math. 220 (2009), 620-656.

[AT1] M. Abate, F. Tovena: Parabolic curves in $\mathbb{C}^{3}$. Abstr. Appl. Anal. 2003 (2003), 275-294.

[AT2] M. Abate, F. Tovena: Poincaré-Bendixson theorems for meromorphic connections and homogeneous vector fields. To appear in J. Diff. Equations. ArXiv:0903.3485, 2011.

[BB] P. Baum and R. Bott: Singularities of holomorphic foliations. J. Diff. Geom. 7 (1972), 279-342.

[FS] J.-E. Fornæss, N. Sibony: Complex dynamics in higher dimension. I. Astérisque 222 (1994), 201-231.

[H] R. Hartshorne: Residues and duality. Lecture Notes in Math. Vol. 20, Springer, Berlin, 1966.

[K] D.E. Knuth: Fundamental algorithms. Addison-Wesley, Reading, MA, 1973.

[L] D. Lehmann: Résidues des sous-variétés invariants d'un feuilletage singulier. Ann. Inst. Fourier, Grenoble 41 (1991), 211-258.

[LS] D. Lehmann and T. Suwa: Residues of holomorphic vector fields relative to singular invariant subvarieties. J. Diff. Geom. 42 (1995), 165-192.

[Su] T. Suwa: Indices of vector fields and residues of singular holomorphic foliations. Hermann, Paris, 1998.

[U] T. Ueda: Complex dynamics on projective spaces - index formula for fixed points. In Dynamical systems and chaos, Vol. 1 (Hachioji, 1994), World Sci. Publ., River Edge, NJ, 1995, pp. 252-259. 